\title{
Changes in egg buoyancy during development and its effects on the vertical distribution of anchovy eggs
}

\author{
Andrés Ospina-Alvarez ${ }^{\mathrm{a}, *}$, Isabel Palomera ${ }^{\mathrm{a}}$, Carolina Parada ${ }^{\mathrm{b}}$ \\ ${ }^{a}$ Institut de Ciències del Mar (CSIC), Passeig marítim de la Barceloneta 37-49, CP \\ 08003, Barcelona, Spain. \\ ${ }^{b}$ Instituto de Investigación Pesquera (INPESCA), Concepción, Chile
}

\begin{abstract}
Small pelagic fish populations exhibit reproductive strategies resulting from past natural selection pressure, by which certain traits become more or less common in a population, allowing them to adapt and become better suited to certain habitats. One such adaptation is the buoyancy of eggs, which is observed as density changes during development. This is an important issue in fisheries and modeling science, as it affects the vertical distribution of eggs and, therefore, egg transport. Recently, individual-based models for anchovies in the Mediterranean have focused on developing adequate biological algorithms to simulate realistic spatial variations of eggs and larvae. Some models that include movements of particles according to Stokes law also assume a constant value of egg density during egg development. However, field observations show differences in the vertical distribution of eggs when egg density during development is considered. We address the problem of egg
\end{abstract}

\footnotetext{
*Corresponding author Email addresses: aospina.co@gmail.com (Andrés Ospina-Alvarez ), isabel@icm.csic.es (Isabel Palomera), cparada@inpesca.cl (Carolina Parada) $U R L:$ http://andresospina.tumblr.com/ (Andrés Ospina-Alvarez )
} 
density and its vertical distribution within a biological context. In Mediterranean waters, the incubation time for anchovy eggs during peak spawning is approximately 48-70 h; during these first hours, egg density has an influence on the horizontal and vertical trajectories of eggs, as well as their routes and hatching zones. In this study, we introduce an algorithm describing the egg density of European anchovy eggs throughout development. Egg density measurements were carried out in a density gradient column (DGC). We fitted a polynomial model that estimated egg density, as a function of time from fertilization and that was dependent on temperature. Simulations to study the vertical transport of eggs in the Mediterranean were carried out using ICHTHYOP/MARS3D. The vertical distribution of pelagic eggs was determined by a set of interacting biological and physical parameters related to eggs (density, diameter) and ambient seawater (density, viscosity, turbulence), respectively. The egg buoyancy model introduced here was validated and will provide insight for the design of anchovy egg surveys, as the vertical position of the eggs in the water column during development can be inferred by the hydrographic structure of seawater.

Keywords: European anchovy, Egg buoyancy, Egg density, Vertical distribution, IBM, Individual based model, DVM, CUFES

\section{Introduction}

The individual-based modeling (IBM) approach is based on the derivation of the properties of ecological systems, such as populations, emerging from the properties of the individuals constituting these systems (Lomnicki, 
1992). Additionally, in IBMs, the spatial location of individuals implies local interactions with the environment (Huston and DeAngelis, 1988), which allows studying environment-resource relationships naturally.

Moreover, fitness-seeking adaptation occurs at the individual level, not at population levels (Grimm and Railsback, 2005). In this context, small pelagic fish are not expected to adapt their egg buoyancy to maximize the persistence of their population. Instead, population-level properties (e.g., patterns of abundance over space and time, mortality and survival) emerge from the interactions between adaptive individuals and their environment, such as properties related to currents, river plumes, sea temperature and salinity.

\subsection{Gulf of Lions: observations}

The Gulf of Lions (GoL), located in the Southern France, is the most important continental shelf in the North-western Mediterranean Basin in terms of circulation. The Northern Current (NC), wind driven up and downwellings (Hua and Thomasset, 1983; Nihoul, 1982), inertial phenomena (Millot, 1990) and discharges from the Rhone River drive the circulation over the shelf. The $\mathrm{NC}$ is mainly composed of Modified Atlantic Water (MAW) at the surface and Levantine Intermediate Water (LIW) from the eastern basin just below the surface. In summer, the NC is relatively wide and shallow, whereas in winter, it becomes narrower and deeper, and tends to flow closer to the slope. Under specific shallow stratification and wind conditions, a branch of the $\mathrm{NC}$ can intrude into the GoL across the eastern boundary (Echevin et al., 2003). In summer, Tramontane and Mistral winds are highly transient in speed with a short lifetime (a few days), and they trigger upwelling events that can easily 
be detected in this season (André et al., 2005).

\subsection{The importance of the buoyancy of fish eggs}

Spawning patterns are a fundamental issue for understanding fish-stock variability in the context of life-history strategies (Aoki and Murayama, 1993). The environmental conditions that prevail during spawning could have an important influence on the survival of eggs and larvae. Populations of small pelagic fish exhibit reproductive strategies resulting from past natural selection pressure, allowing them to adapt to high habitat variability (Brochier et al., 2009). One such adaptation is changes in egg buoyancy due to density changes during development. This adaptation leads to the appearance of different life histories when processes such as egg advection, transport, retention variability, or egg incubation and hatching time are considered in relation to egg buoyancy.

The vertical position of pelagic fish eggs and larvae in the water column determines (i) the extent and direction in which they might be displaced or transported, (ii) their development rates and (iii) mortality, depending on the overlap with their predators, ambient food and physiochemical conditions; and these three factor condition impacts on (iv) recruitment success (Page et al., 1989; Parada et al., 2003; Sundby, 1997). Knowledge of the factors influencing the vertical distribution of fish eggs is a crucial issue in fisheries science (Goarant et al., 2007). Survival models, egg production methods, transport and drift models and recently developed coupled hydrodynamicIBMs are sensitive to the initial conditions of eggs in the water column.

In the past 13 years, the use of the continuous sampler CUFES (Continuous Underway Fish Egg Sampler; Checkley et al., 1997) has been increas- 
ingly adopted to reduce sampling time during Daily Egg Production Method (DEPM surveys; Lasker, 1985; Checkley et al., 2000). The major limitation of CUFES is that it collects samples at a fixed depth $(3 \mathrm{~m})$. However, the vertical distribution of fish eggs in the water column changes and, in some cases, generates considerable concentrations at depths below $5 \mathrm{~m}$ (as with European anchovy in the Mediterranean; Olivar et al., 2001). Therefore, CUFES abundance estimates can be biased depending on the species and the water density conditions sampled.

Stokes law mainly determines the terminal velocity of the pelagic eggs. Egg vertical distribution results from the interaction of the biological and physical parameters of gravitational acceleration, seawater density, seawater viscosity, mixing and turbulence, as environmental parameters, and egg density, egg radius and egg shape, as biological parameters (Solemdal and Sundby, 1981; Sundby, 1997). Field experiments are complex, and horizontal flow field variability with depth is considerable (Sundby, 1997). Coombs (1981) introduced the density gradient column (DGC) to measure the neutral buoyancy of fish eggs. Subsequently, models for many species have been developed using the DGC (Boyra et al., 2003; Coombs et al., 1985; Haug et al., 1986; Tanaka, 1990), with some limitations linked to limited biological knowledge (Goarant et al., 2007).

Most marine fishes spawn pelagic eggs that are externally fertilized and float individually near the surface (Kendall et al., 1984). The osmotic consequences for a teleost embryo when developing in freshwater are water influx and continuous ion loss (Fyhn et al., 1999). In contrast, when teleost embryos develop in seawater, water continuously flows into the environment, 
and environmental ions tend to diffuse into the egg (Fyhn et al., 1999). As an adaptation to life in hyperosmotic seawater, pelagic marine teleost eggs have a large reservoir of yolk water, with water content accounting for 9296\% of the egg wet mass at spawning time (Craik and Harvey, 2009; Fyhn et al., 1999). Hydration occurs during the reinitiation of meiosis (oocyte maturation) just prior to ovulation, and this process determines the initial egg density at spawning (Fabra et al., 2005; Fulton, 1898; Wallace and Selman, 1981). Oocyte hydration involves free amino acids derived from yolk proteolysis, which result in increasing oocyte osmolarity (Craik and Harvey, 2009; Fyhn et al., 1999) and water passage through the vitelline membrane via molecular water channels (Fabra et al., 2005). Thus, spawning adults adjust the initial egg density to the environmental conditions prior to spawning, and subsequently, egg membranes may be subjected to environmental modification (Laale, 1980). This phenomenon may involve effective osmoregulatory mechanisms that adjust the specific gravity of eggs in accordance with the surrounding seawater environment (Holliday and Blaxter, 1960).

Based on the above findings, we introduced an IBM that accounts for egg buoyancy changes during development. Our model focuses on the vertical distribution of eggs and considers both horizontal and vertical transport in the GoL. Buoyancy is highlighted as an important biological characteristic that influences the egg position in the water column. Model outputs were compared with data on egg vertical distribution described in previous studies. Finally, we discuss our results regarding the use of CUFES as a sampling method for DEPM surveys. 


\section{Methods}

Samples were collected in June 2008 onboard the RV Garcia del Cid during the MPOCAT 08/ICM-CSIC survey. Eggs and early larval stages are located in the surface layers of the water column, above the thermocline (Palomera, 1991), with 90\% of eggs found in the upper $15 \mathrm{~m}$ (Olivar et al., 2001). Therefore, vertical hauls were carried out using a 1-m mouth diameter net with a 150- $\mu \mathrm{m}$ mesh size at a maximum depth of $50 \mathrm{~m}$. Egg samples were immediately transferred to $1 \mathrm{~L}$ containers of local filtered seawater and taken to the laboratory onboard. Anchovy eggs and their stages were identified with a stereoscopic microscope and introduced (one by one) into a DGC (Coombs, 1981; Coombs et al., 2004). The complete process from sampling to the introduction of the eggs into the DGC took less than thirty minutes. The DGC was kept at a thermostatic controlled temperature of $18.7 \pm 0.2^{\circ} \mathrm{C}$. The density in the columns used in the experiments ranged from approximately 21.3 (at the top) to 28.5 sigma-t (at the bottom). The DGC can resolve differences in density of 0.04 sigma-t at maximum accuracy (Coombs, 1981). The height of eggs in the column is an indication of their density (the higher the position in the column, the lower the density), and height was measured at 1.5-h intervals until each individual egg hatched.

The main group of eggs studied was collected at 2:15 am from station A $\left(43^{\circ} 12,108 \mathrm{~N} 4^{\circ} 31,617 \mathrm{E}\right)$, when a considerable amount of early stages (I, II) were sampled (Fig. 5.1). Eggs from B-D stations were taken at night or at dawn. Nevertheless, eggs from these stations were not primarily early stages and so were used in the complementary DGC experiments. 


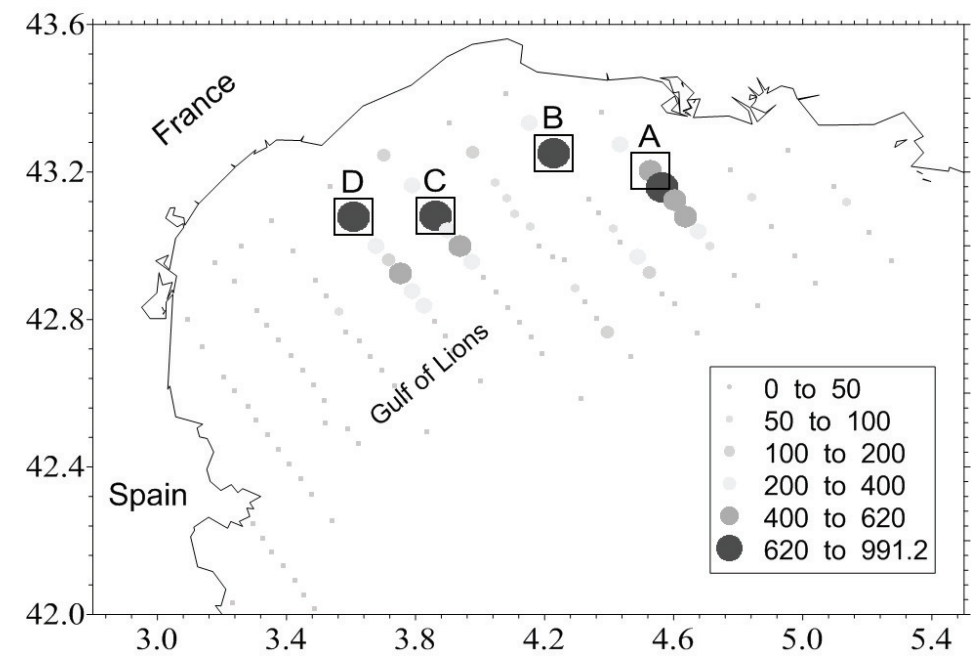

Figure 1: Composite distribution map of anchovy eggs collected using a CalVET net during DEPM surveys in 2008. The colour and size of the circles show the relative abundance in eggs $\times m^{-2}$. Zones A-D, where anchovy eggs were surveyed for the DGC experiments, are indicated. 


\subsection{Model formulation}

The total incubation time of anchovy eggs increases with decreasing temperature. Moreover, the duration of each egg stage (I-X) is not constant; some stages are longer than others.

We used a power function (Regner, 1985) for estimating the total developmental time:

$$
\text { DevTime }=C \times T^{(-b)}
$$

where DevTime is the total development time; $\mathrm{C}$ and b are constants; and $\mathrm{T}$ is the temperature in ${ }^{\circ} \mathrm{C}$. For anchovies, we used the following values for $\mathrm{C}$ and $\mathrm{b}$ :

$$
\text { DevTime }=42,922.0767 \times T-2.290236
$$

Although the $\mathrm{C}$ and $\mathrm{b}$ values in Eq. (2) were calculated based on the method of (Regner, 1985) for anchovies in the Adriatic Sea, these constants correctly predict the hatching time for anchovies in Mediterranean and Cantabrian waters, and their use is broadly accepted in DEPM and otolith growth studies (Aldanondo et al., 2008; Palomera and Pertierra, 1993; Somarakis et al., 2004). In our DGC experiment at $18.7^{\circ} \mathrm{C}$, the DevTime required for hatching was $52.5 \mathrm{~h}$.

We obtained the $\mathrm{C}$ value for each experimental time step using exponent laws:

$C=D \times T^{2.290236} \quad$ subsequently, $C i=($ time from spawning $) \times T^{2.290236}$ 
Table 1: Developmental constant (C), developmental ratio and residence time factors per egg stage needed to determine an egg buoyancy equation for anchovy eggs. Calculations are based on the Regner equation (1985).

\begin{tabular}{rllcc}
\hline Stage & C value for each stage & Developmental ratio & $\begin{array}{l}\text { Percentage of residence } \\
\text { time in each stage }\end{array}$ & $\begin{array}{c}\text { Accumulative percentage } \\
\text { of residence time }\end{array}$ \\
\hline 2 & 6953.38 & 0.16 & 16.30 & 16.30 \\
3 & $10,515.91$ & 0.25 & 7.95 & 24.25 \\
4 & $16,954.22$ & 0.40 & 15.06 & 39.31 \\
5 & $20,430.91$ & 0.48 & 8.37 & 47.68 \\
6 & $28,714.87$ & 0.67 & 19.25 & 66.93 \\
7 & $33,393.38$ & 0.78 & 11.73 & 78.66 \\
8 & $38,200.65$ & 0.89 & 10.46 & 89.12 \\
9 & $40,904.74$ & 0.95 & 6.28 & 95.40 \\
10 & $42,922.08$ & 1.00 & 4.60 & 100.00 \\
\hline
\end{tabular}

Then, we calculated the developmental ratio (DevRat) for each experimental time:

$$
\text { DevRat }=\frac{C i}{C}
$$

Table 5.1 summarizes our calculations for the developmental constant (C), developmental ratio and residence time factors per anchovy egg stage, as derived from studies by (Regner, 1985, 1996).

Then, based on Eq. (4) and the egg density values obtained from the DGC, we fit a polynomial equation for estimating egg density considering the time from fertilization, the effect of temperature, density at spawning ( $\mathrm{SpD}$ ) and density at hatching (HtD). Subsequently, a general model to calculate the egg density changes during development in the GoL was proposed.

\subsection{The anchovy egg transport simulations}

The egg density model obtained was programmed into ICHTHYOP IBM software v.3 (Lett et al., 2008), modifying the original eggs buoyancy scheme that assumed a fixed egg density during development. ICHTHYOP was de- 
veloped to study how physical (e.g., ocean currents and temperature) and biological (e.g., buoyancy and growth) factors affect the dynamics of ichthyoplankton using time series of velocity, temperature and salinity fields obtained from oceanic simulations of the Model for Applications at Regional Scale (MARS; Lazure and Dumas, 2008). It also enables the tracking of virtual drifters and the ocean properties (e.g., temperature, salinity and density) encountered by virtual particles (Fig. 5.2).

In this study, we focused on the vertical movement of eggs. A 3D approximation is necessary to study the seawater changes affecting egg transport and development. Thus, to simulate horizontal transport, we added movement to simulated particles in the IBM MARS-3D output. In the IBM, vertical movement resulted from the modeled hydrodynamic vertical field and the terminal velocity was calculated based on the characteristics of each particle (density, diameter, gravitational force, vertical eddy diffusivity, seawater density and viscosity).

Three types of simulation experiments were conducted: (1) Pure Lagrangian experiments (hereafter, virtual particle); (2) Lagrangian with constant egg density experiments, using the seawater $\mathrm{SpD}$ as the egg density value (hereafter, constant egg buoyancy experiments), and (3) Lagrangian with variable egg density experiments (hereafter, variable egg buoyancy experiments).

For NW Mediterranean and Bay of Biscay (BoB) anchovies, in situ observations have revealed that adult fish ascend to surface waters at night and descend to deeper waters at dawn during spawning (Masse, 1996; Palomera, 1991). According to Palomera and Lleonart (1989), the European anchovy 

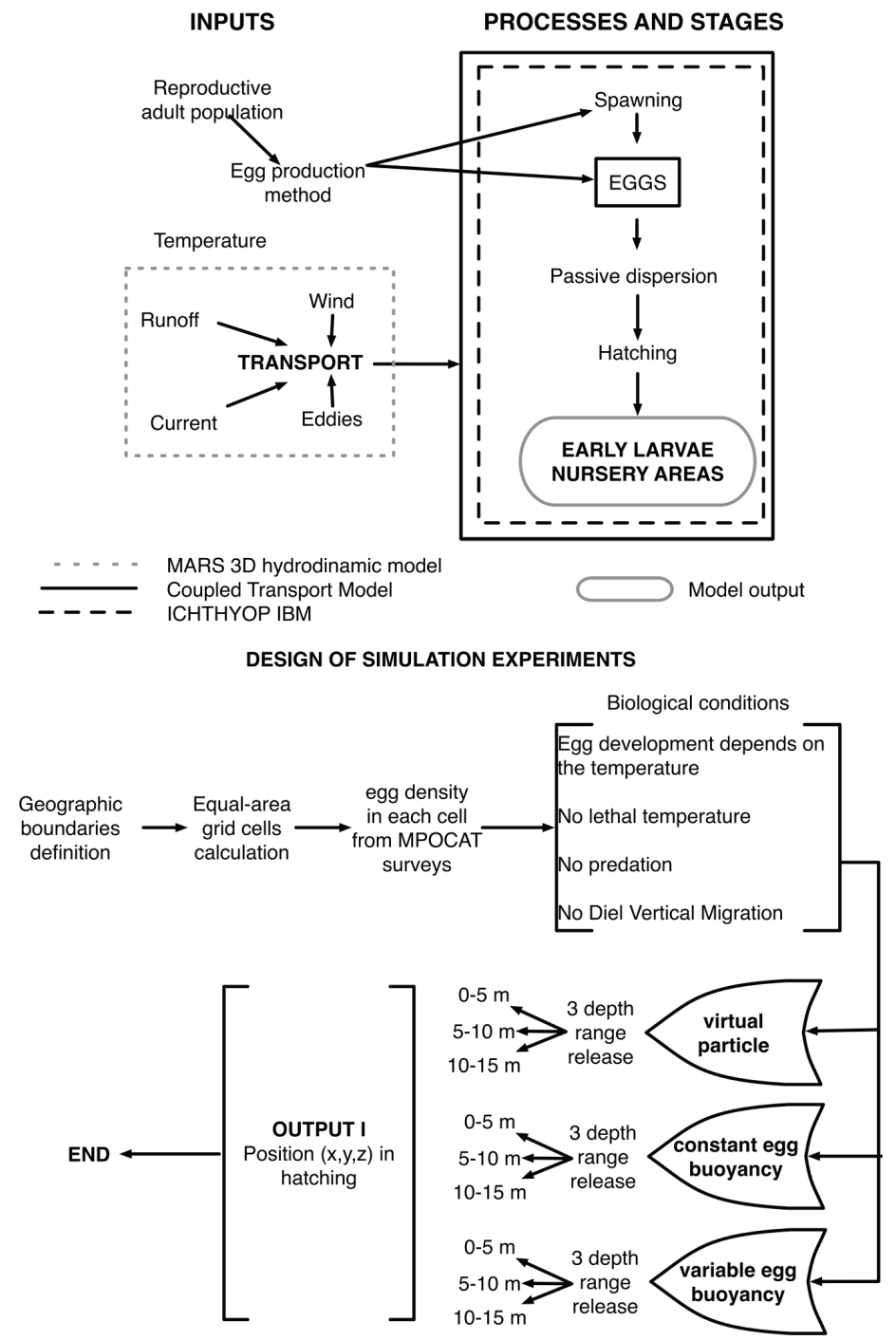

Figure 2: Simplified scheme of model coupling shows physical forcings (wind, runoff, currents), biological process (spawning, hatching) and modeled inputs and outputs (above). Design of the simulation experiments indicating biological conditions, types of experiments and modeled outputs (below). 
spawns from 20:00 h to 4:00 h GMT at night, with a peak around midnight. The depths observed for early egg stages show that anchovies spawn close to the surface at depths of $<10 \mathrm{~m}$ (Olivar et al., 2001). However, we initiated the experiments (virtual particle, constant egg buoyancy and variable egg buoyancy) with differential depth ranges (0-5 m, 5-10 m and 10-15 m) to test the influence of the spawning depth on vertical transport. We performed nine experiments.

Field egg data were used to establish an initial number of particles released per quadrant in each experiment. A total of 20,671 particles representing a fraction of the $2.0671 \times 10^{12}$ anchovy eggs estimated from field data were virtually released in the GoL in each experiment (Fig. 5.1). The number per quadrant is variable and represents the field egg abundance. The release time in each experiment was midnight on the 6th of June 2008. As the total incubation period of anchovy eggs varies with temperature, we stopped the movement of each particle (egg) when it reached hatching (48-70 h).

\section{Results}

The GoL is hydrodynamically a very complex region due to (i) the general southwestward circulation along the slope (NC); (ii) the wind-induced currents; and (iii) the formation of dense water, both on the shelf and offshore (Millot, 1990). Seasonal variation of the stratification of the surface layers is a hydrological characteristic of the GoL (Millot, 1990). The thermocline, which is also a pycnocline, is important from a dynamic point of view. It allows the slipping of the surface layer over the bottom layer, with each layer behaving independently, and thus, the vertical shear is reduced (Millot, 
1990). During the MPOCAT 08/ICM-CSIC survey, the vertical structure of the water column was observed to exhibit typical summer thermal stratification (Fig. 5.3).
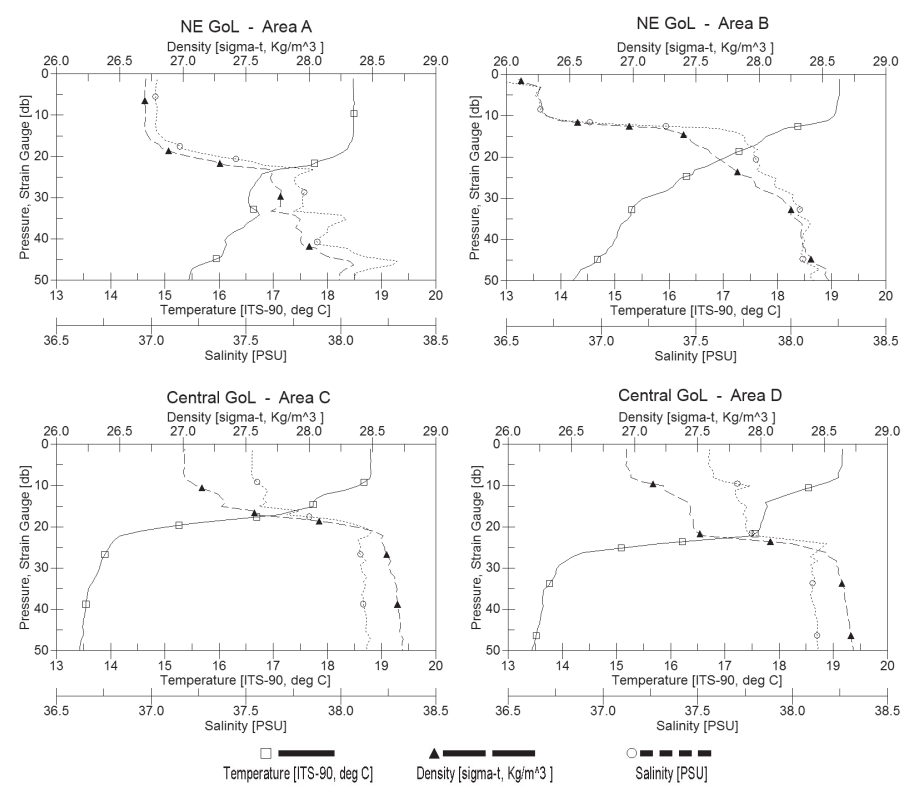

Figure 3: Mean vertical profiles of temperature in ${ }^{\circ} \mathrm{C}$ (squares), density in sigma-t or $\mathrm{kg}$ $\times m^{3}$ (triangles) and salinity in psu (circles) for stations A-D, where eggs were surveyed for experiments in June 2008.

The freshwater input in the study area is mainly controlled by the Rhone River, which is the largest source of freshwater in the Mediterranean Sea, representing $92 \%$ of the input into the GoL (Bourrin et al., 2006). Consequently, stations A and B, with lower salinities, clearly show the influence of Rhone River outflow. The difference between the seawater density at the surface and at the pycnocline depth was between 1.2 and 1.5 sigma-t units for the four stations. (Goarant et al., 2007) found a positive correlation between egg density and seawater salinity. We found that anchovy egg density was 
also correlated with the seawater salinity and density at the surface in the GoL (Fig. 5.4).

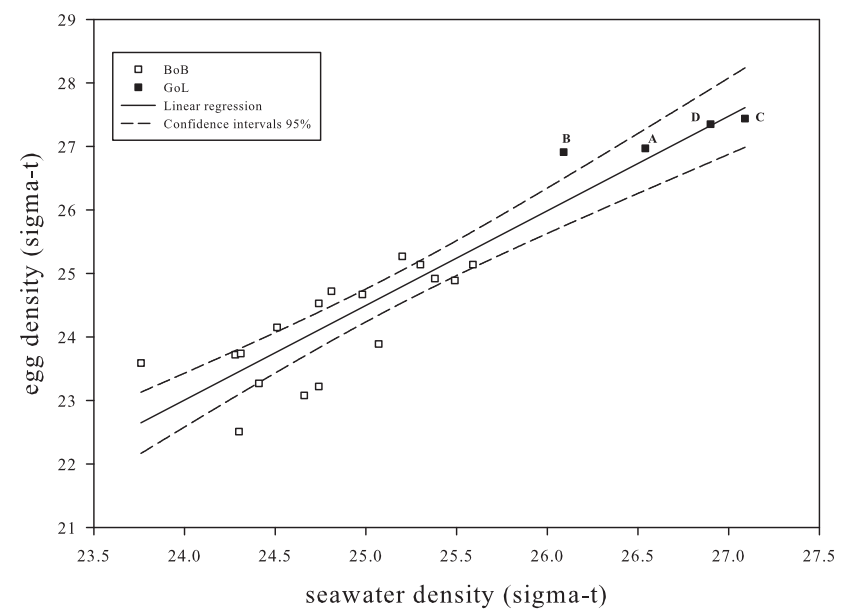

Figure 4: Regression of mean anchovy egg density (sigma-t) with sea surface (3m) density in the $\mathrm{BoB}$ (white squares, adapted from Goarant et al. 2007 and GoL (black squares, this study). Stations A-D are labeled. Dotted lines indicate the $95 \%$ confidence interval for the mean egg density. The fitted line is the linear regression: eggdensity $=-12.7675+$ $1.4906 \times($ seawater density $), r^{2}=0.8555$.

\subsection{Egg density measurements}

Based on the DGC experiments, the egg density at spawning time was found to be lower than at hatching time (Fig. 5.5). The main group of eggs used in the DGC experiments corresponded to no-embryo eggs from station A. In the main experiment, using eggs from station A, the mortality prior to hatching was $19.2 \%$ (5 eggs from 26 introduced into the DGC). The results from the DGC experiments with eggs from station B (41 eggs, 
mainly of stages IV-VI, mortality 14.6\%); C (37 eggs, mainly of stages VVIII, mortality 21.6\%) and D (34 eggs, mainly of stages III-V, mortality $26.5 \%$ ) were used to verify the results of the main DGC experiment. All DGC experiments showed that egg density followed a pattern during development that depended on the characteristics of the seawater in which the eggs were spawned.

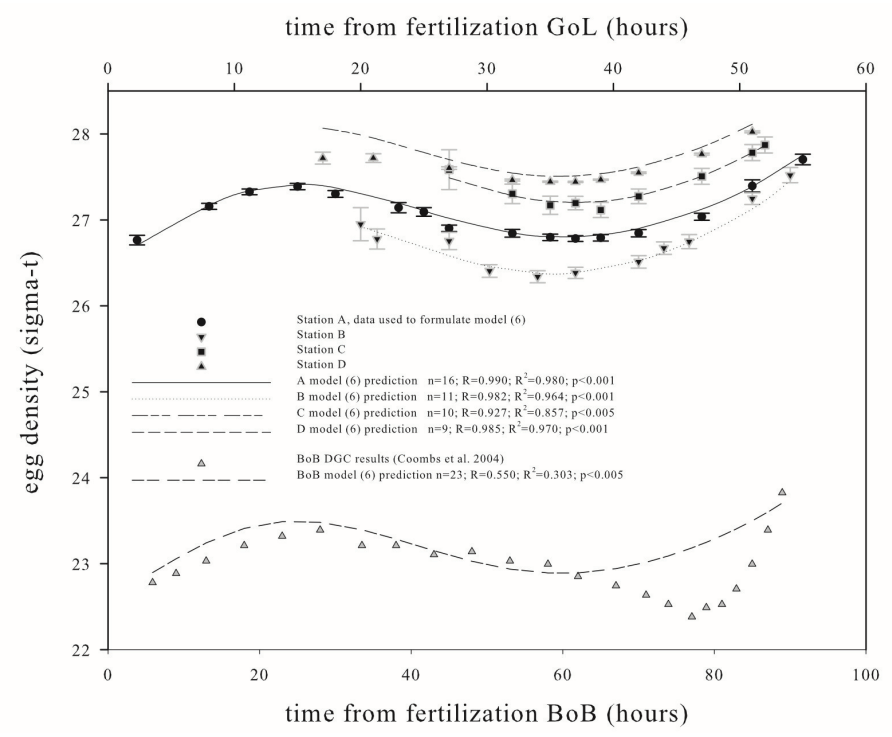

Figure 5: Changes in median anchovy egg density during development at stations A (circles, up), B (triangles, down), C (squares) and D (triangle up). The lines are predictions from the egg-buoyancy model (Eq. (5.6)). The GoL and BoB are represented at different scales due to the effect of temperature on development.

\subsection{A model for egg density during development}

Egg density can be easily inferred considering the time from fertilization, the incubation temperature, SpD and HtD. 


$$
\begin{aligned}
& \text { Egg density }=\left(41.568 \times \text { DevRat }^{6}-149.788 \times \text { DevRat }^{5}\right. \\
& \text { +200.864 × DevRat }{ }^{4}-114.050 \text { DevRat }^{3} \\
& \left.+19.789 \times \text { DevRat }^{2}+2.423 \times \text { DevRat }\right) \\
& \times(H t D-S p D)+S p D
\end{aligned}
$$

However, we sought to verify whether Eq. (5) could be generalized to (i) other zones in the GoL and (ii) other regions, such as the BoB. In the first case, we required a general approximation because the seawater density was not known at the hatching time for eggs from any stations or at spawning time for eggs from B-D stations. Then, taking into account that mean egg density displays a significant positive correlation with sea surface salinity (Goarant et al., 2007) (Fig. 5.4), we calculated the equivalent seawater density at the spawning and hatching times using SpD and HtD, respectively, from the DGC experiment results using eggs from station A. The difference between the initial egg density and the final egg density was approximately 1.2 sigma$\mathrm{t}$ units. This value corresponds to the mean difference between the seawater density at the surface and the seawater density at the pycnocline depth at stations A-D (Fig. 5.3). Model (6), which was developed from Eq. (5) using eggs from station A, correctly predicts the egg density changes during development for eggs from the other GoL stations (Fig. 5.5).

In the second case, for a generalization of Eq. (5) to other zones, we calculated DevTime $(86.9 \mathrm{~h})$ for anchovy eggs based on the results from experiments in the $\mathrm{BoB}$ at $15^{\circ} \mathrm{C}$ (Coombs et al., 2004) using Eq. (2). This equation is suitable for predicting DevTime for anchovy eggs in Cantabrian waters (Aldanondo et al., 2008), as in the Adriatic (Regner, 1985) and the NW Mediterranean (Palomera and Pertierra, 1993). To compare the GoL 
and BoB DGC experiments, we eliminated BoB observations greater than DevRat $=1$. An interesting observation emerged from this comparison: the difference between $\mathrm{SpD}$ and $\mathrm{HtD}$ was approximately 1.2 sigma-t units in both the GoL and BoB zones. Given this similarity, a general model to calculate egg density changes during development could be proposed, eliminating the necessity of recording the seawater density at hatching time:

$$
\begin{gathered}
\text { Egg density }=49.882 \times \text { DevRat }^{6}-179.746 \times \text { DevRat }^{5} \\
+241.037 \times \text { DevRat }^{4}-136.860 \times \text { DevRat }^{3} \\
+23.747 \times \text { DevRat }^{2}+2.908 \\
\times \text { DevRat }+ \text { SpD }
\end{gathered}
$$

Thus, model (6) only needs to include the DevTime, the incubation temperature and the seawater density at spawning, which is equivalent to $\mathrm{SpD}$. Predictions from egg density model (6) were compared with the DGC data for both zones. The modeled curves obtained are a good representation of both experiments, validating our model for the Gulf of Lions and the Bay of Biscay (Fig. 5.5).

\subsection{Modeling anchovy egg transport during development}

The virtual particle and constant and variable egg buoyancy experiments are horizontal and vertical transport simulations. The virtual particle experiments do not include an estimation of egg density; and the constant egg buoyancy experiments considered the egg density as a constant value throughout development, which corresponds to the seawater density at spawning. Model (6) was only included in variable egg buoyancy experiments. The depth during development depends on the release depth in all experiments. In the 
virtual particle experiments, the particles remain at the released depths. In the constant egg buoyancy experiments, the particles are either positively buoyant or not, depending on the release depth; for example, in the 0-5 m range, particles moved toward the surface. The particles in the variable egg buoyancy experiments tended to accumulate near the pycnocline; particles released in the 0-5 $\mathrm{m}$ range traveled a longer distance in the vertical profile than particles released at either the $5-10 \mathrm{~m}$ or $10-15 \mathrm{~m}$ ranges (Fig. 5.6).
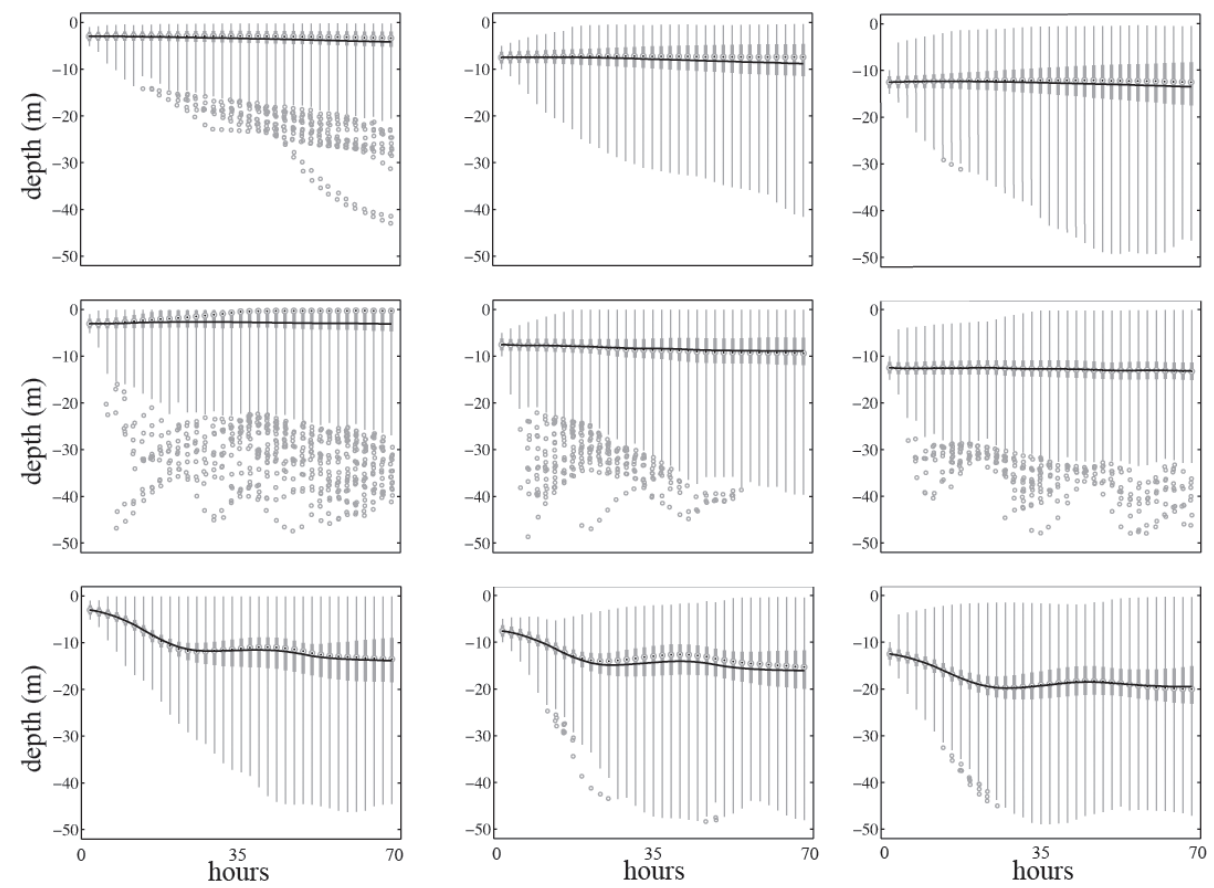

Figure 6: MARS-3D-ICHTHYOP coupled model output. Egg depth (meters) during development in virtual particle (above), constant egg buoyancy (centre) and variable egg buoyancy experiments (bottom). The graphs correspond to 0-5 m on the left, 5-10 m in the middle and 10-15 $\mathrm{m}$ on the right. The graphs indicate the mean values (dark lines) for egg-buoyancy and the egg-depth distributions of the simulations throughout egg development (median, first and third quartiles, max, min and extreme values). 
Grouping the results from all variable egg buoyancy experiments, we observe that the egg concentration at hatching was higher when near to the pycnocline than when near to the surface or deep waters. The main concentration throughout development occurred between 10.0 and $20.8 \mathrm{~m}$. The egg depths were mainly concentrated between 4.0 and $11.2 \mathrm{~m}$ at spawning and between 13.0 and $23.0 \mathrm{~m}$ at hatching (Fig. 5.7).

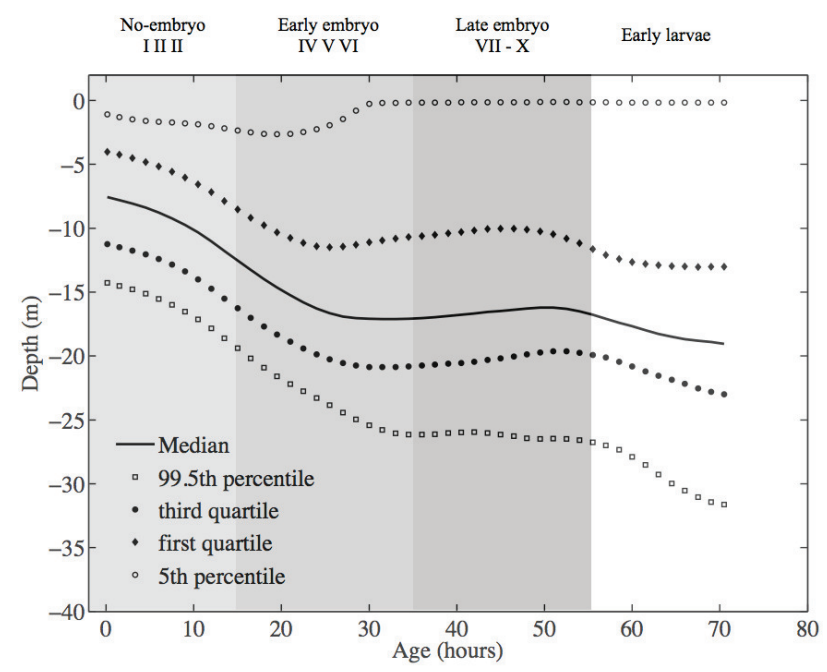

Figure 7: MARS-3D-ICHTHYOP coupled model output with the pull of depth ranges in the variable egg buoyancy experiment. The graph shows the vertical distribution of eggs (no-embryo, early and late embryo) and early larvae during development, including the median, 1st and 3rd quartiles and 5th and 99.5th percentiles.

\section{Discussion}

One of the main features of IBMs is that the spatial location of individuals implies local interactions with the environment (Huston and DeAngelis, 1988). This study, which was based on an IBM approach, mainly focused on 
the influence of egg buoyancy in each developmental stage to understand the vertical distribution of eggs. Previous studies on ichthyoplankton transport have ignored changes in egg buoyancy. Our results showed that the variability of egg buoyancy as a function of development is significant with respect to vertical transport.

The main spawning areas for anchovies in 2008 in the GoL showed a clear influence of the Rhone River: north (A) 36.96 psu and (B) 36.63 psu, in contrast with central area (C) 37.53 psu and (D) 37.55 psu (Figs. 5.1 and 5.3). The sea surface salinities at sites where the main groups of eggs for the DGC experiments were collected were higher in the GoL (36.96 psu) than in the BoB (34.60 psu; Coombs et al., 2004). Moreover, the relationship between egg density and seawater salinity in the GoL was linear, in accordance to with the findings of Goarant et al. (2007). A sigmoid-like relationship, in which egg density would reach low and high sills at low and high salinities respectively, as was suggested in the BoB study (Goarant et al., 2007), was not found in the GoL, although this might be expected to occur at higher salinities.

Under the experimental conditions employed in the DGC, the egg density (no embryo, stages I-II) was slightly lower than the surrounding seawater density in the NW Mediterranean. The egg density changes during development followed a similar pattern in the four stations in the GoL and in the $\mathrm{BoB}$, that is, increasing by 1.2 sigma-t units from spawning until hatching. However, egg densities were clearly different between the BoB and GoL due to the seawater salinity (Goarant et al., 2007) and the oceanographic characteristics influencing physicochemical egg development (Holliday and Blaxter, 1960). Model (6) provided a statistically good fit with the GoL and the BoB 
DGC experiments. This could improve the predicted vertical and horizontal trajectories from transport-IBM coupled models.

The MARS-3D-ICHTHYOP coupled model, with the added model (6), reproduced the field variability in egg vertical distribution through development well, with eggs appearing within a wide range of water column depths, but being concentrated in surface layers. In the NW Mediterranean, simulated data from variable egg buoyancy experiments suggested eggs concentrations above and at the base of the pycnocline at hatching time. Even if eggs were distributed between depths of zero and $50 \mathrm{~m}$, the interval in which they were most likely to be present was between 4.0 and $11.2 \mathrm{~m}$ at spawning and above the pycnocline at hatching. Additionally, spawning and hatching are known to take place in the depth intervals we modeled (Olivar et al., 2001; Sabatés et al., 2008).

DEPM egg survey designs have to be adapted to local population dynamics to optimize the survey results. The precision of egg production estimates is strongly dependent on the correct sampling of all egg stages at sea. The CUFES continuously collects samples in motion at a fixed depth (e.g., $3 \mathrm{~m}$ ), but it has been demonstrated that the eggs are not uniformly distributed throughout the water column, and CUFES sampling is limited to $3 \mathrm{~m}$. Our results showed that only a minimal portion of the anchovy eggs remain in the surface layers after spawning. Some studies have proposed calibrating CUFES with traditional samplers, such as a CalVET or Bongo sampler (Smith et al., 1985), converting CUFES data to egg abundance using a vertical distribution model (Pepin et al., 2007), or combining acoustic and CUFES data (Petitgas et al., 2009). Nevertheless, these studies are based 
on two questionable assumptions: (1) that the same vertical profile exists for all egg categories, i.e., assuming that egg buoyancy does not vary during egg development, and (2) that pelagic fish eggs are positively buoyant throughout the majority of their development, with eggs typically accumulating just below the surface (Coombs et al., 2004).

The first assumption is not always valid because the concentrations of pelagic fish eggs sometimes vary drastically within a half-meter in the vertical direction (Tanaka, 1992), and phytoplankton and zooplankton concentrations fluctuate below, as well as above, $0.2 \mathrm{~m}$ (Owen, 1989). Therefore, we highlight the importance of including these small variations in egg buoyancy when the aim of the study is to understand how ascending/descending velocities due to species-specific and/or developmental stage-specific density, shape and dimensions control the vertical distribution of eggs (Sundby, 1997). Our results concur with those of several studies (Coombs et al., 2004; Curtis et al., 2007; Tanaka, 1990) in showing that pelagic fish eggs change their specific gravity during development.

Regarding the second assumption, Tanaka (1992) found that in the Japanese anchovy, the egg concentration decreased exponentially with depth, and there was a clear accumulation of eggs at the pycnocline. The accumulation of anchovy eggs at the base of the pycnocline has also been observed in Mediterranean waters (Sabatés et al., 2008), and early eggs (no-embryo and stages I-III) are mainly concentrated between 5 and $10 \mathrm{~m}$ (Olivar et al., 2001). Sabatés et al. (2008) found that the egg concentration at the surface (0$10 \mathrm{~m})$ was lower than in the range corresponding to the pycnocline $(16 \pm 3.16$ and $30 \pm 13.54)$ in two different zones with different vertical environmental 
conditions off the Catalan coast.

This would imply that eggs spawned above the pycnocline would have negative buoyancy and tend to sink, while eggs spawned below the pycnocline would have positive buoyancy and tend to rise and eggs spawned near the pycnocline with neutral buoyancy would remain at the same depth. However, the spawning depth has already been revealed to be near the surface, clearly above the pycnocline (Olivar et al., 2001; Sabatés et al., 2008; Sekiguchi and Sugishima, 1995). Therefore, the pycnocline could act as a boundary in the vertical distribution of eggs. We can assume that the density of eggs is adjusted prior to ovulation to values slightly lower than the water below the pycnocline. However, eggs cannot be positively buoyant in seawater of low density in the upper layer (Tanaka, 1992). Additionally, considering the vertical difference in the rising/sinking rates of eggs, estimation of the vertical eddy diffusivity in the surface layer emerges as an important parameter (Pepin et al., 2007). However, some studies use a constant eddy diffusivity value below the mixed layer (Ådlandsvik et al., 2001; Curtis et al., 2007). This is an important issue for further developing hydrodynamic-IBM coupled models for studying ichthyoplankton transport.

In variable egg buoyancy experiments, the eggs tend to accumulate near the pycnocline. Although the eggs apparently exhibit parallel vertical trajectories, the vertical distance traveled in the upper layers (0-5m range) is greater than the vertical distance traveled at either the 5-10 $\mathrm{m}$ or 10$15 \mathrm{~m}$ ranges. The vertical distance traveled differs according to spawning depth due to differences in seawater densities at both spawning and hatching depths. As a consequence, egg depth changes can be either gradual or sudden 
according to the vertical salinity gradient.

Considering the frequency of Mistral and Tramontane wind events in the GoL during the summer, it may be ascertained that water masses near the surface, as well as at the bottom, permanently oscillate horizontally and are displaced vertically during the inertial period. The shear between the two layers will be variable in time as well as the current near the bottom (Millot, 1990). This can explain why the pycnocline was encountered at continuously varying depths at different survey stations. In contrast, in May 2001, the pycnocline in the BoB was found at shallower depths of 8-10 m (Coombs et al., 2004). Therefore, the CUFES underestimation could be more significant in Mediterranean waters than in Atlantic waters because in summer months, the pycnocline in the GoL is deeper than in the BoB. The CUFES is often used as a secondary sampler to reduce costs and to improve the precision of determining egg abundances. Nevertheless, the variability in egg density per stage at different spatial scales must be taken in account when using this sampling gear.

In conclusion, we can simulate egg transport in three ways, with eggs as virtual particles, eggs as particles with constant density and eggs as particles with variable density during development. As virtual particles, the accumulation of eggs on a pycnocline was not observed. As particles with constant density, the accumulation was found to occur conditionally, depending on the chosen egg density value. However, this is likely to be a consequence rather than an effect. Therefore, simulation experiments including constant values of egg density are stationary approaches that should be appropriate in specific situations but are not a general solution to this question. 
Finally, integrated egg buoyancy model with 3D hydrodynamic-IBMs represents a non-stationary approach that explains the egg accumulation on a pycnocline under different scenarios. Studying egg density changes during development is necessary if any reliable estimation of egg advection is to be achieved in transport-IBM coupled models. Furthermore, the results from this study could provide useful advances related to the practical design of DEPM surveys.

\section{Acknowledgements}

This research was conducted within the European project SARDONE (FP6 44294). Egg samples were obtained from the MPOCAT-DEPM surveys financed by Generalitat de Catalunya. A. Ospina-Alvarez benefited from a PhD grant of the JAE program (CSIC). We are indebted with AZTI-Tecnalia for providing us with the Density Gradient Column used to conduct our experiments. Thanks to Anne Elizabeth Mohan for proofreading the English. We are grateful to P. Garreau and A. Nicolle for their support with MARS3D hydrodynamic model outputs, as well as to P. Verley for his assistance in ICHTHYOP code. Finally, the authors gratefully acknowledge the assistance of the captain and crew of the RV Garcia del Cid for their help during the cruise.

\section{References}

Ådlandsvik B, Coombs SH, Sundby S, Temple G. Buoyancy and vertical distribution of eggs and larvae of blue whiting (Micromesistius poutassou): observations and modelling. Fish Res 2001;50:59-72. 
Aldanondo N, Cotano U, Etxebeste E, Irigoien X, Alvarez P, Demurguia A, et al. Validation of daily increments deposition in the otoliths of European anchovy larvae (Engraulis encrasicolus L.) reared under different temperature conditions. Fish Res 2008;93:257-264.

André G, Garreau P, Garnier V, Fraunie P. Modelled variability of the sea surface circulation in the North-western Mediterranean Sea and in the Gulf of Lions. Ocean Dynam 2005;55:294-308.

Aoki I, Murayama T. Spawning pattern of the Japanese sardine Sardinops melanostictus off southern Kyushu and Shikoku, southwestern Japan. Mar Ecol Prog Ser 1993;97:127-134.

Bourrin F, Durrieu De Madron X, Ludwig W. Contribution to the study of coastal rivers and associated prodeltas to sediment supply in the Gulf of Lions (NW Mediterranean Sea). Vie Milieu 2006;56(4):307-314.

Boyra G, Rueda L, Coombs SH, Sundby S, Ådlandsvik B, Santos M, et al. Modelling the vertical distribution of eggs of anchovy (Engraulis encrasicolus) and sardine (Sardina pilchardus). Fish Oceanogr 2003;12(4/5):381395.

Brochier T, Colas F, Lett C, Echevin V, Cubillos L, Tam J, et al. Small pelagic fish reproductive strategies in upwelling systems: A natal homing evolutionary model to study environmental constraints. Prog Oceanogr 2009;83(1-4):261-269.

Checkley DM, Dotson RC, Griffith DA. Continuous, underway sampling of eggs of Pacifc sardine (Sardinops sagax) and northern anchovy (Engraulis 
mordax) in spring 1996 and 1997 off southern and central California. DeepSea Res (2 Top Stud Oceanogr) 2000;47:1139-1155.

Checkley DM, Ortner P, Settle L, Cummings S. A continuous, underway fish egg sampler. Fish Oceanogr 1997;6(2):58-73.

Coombs SH. A density-gradient column for determining the specific gravity of fish eggs, with particular reference to eggs of the mackerel Scomber scombrus. Mar Biol 1981;63:101-106.

Coombs SH, Boyra G, Rueda L, Uriarte A, Santos M, Conway D, et al. Buoyancy measurements and vertical distribution of eggs of sardine (Sardina pilchardus) and anchovy (Engraulis encrasicolus). Mar Biol 2004; 145:959-970.

Coombs SH, Fosh C, Keen M. The buoyancy and vertical distribution of eggs of sprat (Sprattus sprattus) and pilchard (Sardina pilchardus). J mar biol Ass UK 1985;65(2):461-474.

Craik J, Harvey S. The causes of buoyancy in eggs of marine teleosts. J Mar Biol Ass 2009;67(01):169-182.

Curtis K, Checkley DM, Pepin P. Predicting the vertical profiles of anchovy (Engraulis mordax) and sardine (Sardinops sagax) eggs in the California Current System. Fish Oceanogr 2007;16(1):68-84.

Echevin V, Crepon M, Mortier L. Interaction of a coastal current with a gulf: application to the shelf circulation of the Gulf of Lions in the Mediterranean sea. J Phys Oceanogr 2003;33:188-206. 
Fabra M, Raldúa D, Power DM, Deen PMT, Cerdà J. Marine fish egg hydration is aquaporin-mediated. Science 2005;307(5709):545-545.

Fulton T. On the growth and maturation of the ovarian eggs of teleost fishes. Tech. rep., 1898.

Fyhn H, Finn R, Reith M, Norberg B. Yolk protein hydrolysis and oocyte free amino acids as key features in the adaptive evolution of teleost fishes to seawater. Sarsia 1999;84:451-456.

Goarant A, Petitgas P, Bourriau P. Anchovy (Engraulis encrasicolus) egg density measurements in the Bay of Biscay: evidence for the spatial variation in egg density with sea surface salinity. Mar Biol 2007;151:1907-1915.

Grimm V, Railsback SF. Individual-Based Modeling and ecology. Princeton series in theoretical and computational biology. Princeton University Press, 2005.

Haug T, Kjorsvik E, Solemdal P. Influence of some physical and biological factors on the density and vertical distribution of Atlantic halibut Hippoglossus hippoglossus eggs. Mar Ecol Prog Ser 1986;33:207-216.

Holliday F, Blaxter J. The effects of salinity on the developing eggs and larvae of the herring. J mar biol Ass UK 1960;39:591-603.

Hua B, Thomasset F. A numerical study of the effects of coastline geometry on wind-induced upwelling in the Gulf of Lions. J Phys Oceanogr 1983; 13:678-694. 
Huston M, DeAngelis D. New computer models unify ecological theory. BioScience 1988;38(10):682-691.

Kendall A, Ahlstrom EH, Moser HG. Early life history stages of fishes and their characters. 1984.

Laale H. The perivitelline space and egg envelopes of bony fishes: a review. Copeia 1980;2:210-226.

Lasker R. An egg production method for estimating spawning biomass of pelagic fish: application to the Northern anchovy, Engraulis mordax. U.S. Department of Commerce, NOAA Technical Report NMFS-36, Wash. DC, 1985.

Lazure P, Dumas F. An external-internal mode coupling for a 3D hydrodynamical model for applications at regional scale (MARS). Adv Water Resour 2008;31:233-250.

Lett C, Verley P, Mullon C, Parada CE, Brochier T, Penven P, et al. A Lagrangian tool for modelling ichthyoplankton dynamics. Envir Model Softw 2008;23:1210-1214.

Lomnicki A. Population ecology from the individual perspective. In DeAngelis D, Gross LJ, editors, Individual-based models and approaches in ecology, pp. 3-17. New York: Chapman and Hall, 1992;.

Masse J. Acoustic observations in the Bay of Biscay: schooling, vertical distribution, species assemblages and behaviour. Sci Mar 1996;60(S2):227234. 
Millot C. The Gulf of Lions' hydrodynamics. Cont Shelf Res 1990;10(911):885-894.

Nihoul JCJ. Hydrodyamics of semi-enclosed Seas. Elsevier Science Ltd, 1982.

Olivar M, Salat J, Palomera I. Comparative study of spatial distribution patterns of the early stages of anchovy and pilchard in the NW Mediterranean Sea. Mar Ecol Prog Ser 2001;217:111-120.

Owen R. Microscale and finescale variations of small plankton in coastal and pelagic environments. J Mar Res 1989;47:197-240.

Page FH, Frank KT, Thompson KR. Stage dependent vertical distribution of haddock (Melanogrammus aeglefinus) eggs in a stratified water column: observations and model. Can J Fish aquat Sci 1989;46(S1):55-67.

Palomera I. Vertical distribution of eggs and larvae of Engraulis encrasicolus in stratified waters of the western Mediterranean. Mar Biol 1991;111:3744 .

Palomera I, Lleonart J. Field mortality estimates of anchovy larvae, Engraulis encrasicolus, in the western Mediterranean. J Fish Biol 1989;35(A):133138.

Palomera I, Pertierra J. Anchovy biomass estimate by the daily egg production method in 1990 in the western Mediterranean Sea. Sci Mar 1993; $57(2-3): 243-251$.

Parada CE, van der Lingen C, Mullon C, Penven P. Modelling the effect of buoyancy on the transport of anchovy (Engraulis capensis) eggs from 
spawning to nursery grounds in the southern Benguela: an IBM approach. Fish Oceanogr 2003;12(3):170-184.

Pepin P, Curtis K, Snelgrove PVR, de Young B, Helbig J. Optimal estimation of catch by the continuous underway fish egg sampler based on a model of the vertical distribution of American plaice (Hippoglossoides platessoides) eggs. ICES J Mar Sci 2007;64:18-30.

Petitgas P, Goarant A, Masse J, Bourriau P. Combining acoustic and CUFES data for the quality control of fish-stock survey estimates. ICES J Mar Sci 2009;66:1384-1390.

Regner S. Ecology of planktonic stages of the anchovy, Engraulis encrasicolus (Linnaeus, 1758), in the central Adriatic. Acta Adriática 1985;26(1):1-113.

Regner S. Effects of environmental changes on early stages and reproduction of anchovy in the Adriatic Sea. Sci Mar 1996;60(S2):167-177.

Sabatés A, Zaragoza N, Grau C, Salat J. Vertical distribution of early developmental stages in two coexisting clupeoid species, Sardinella aurita and Engraulis encrasicolus. Mar Ecol Prog Ser 2008;364:169-180.

Sekiguchi H, Sugishima H. Fine-scale spatial distribution of anchovy eggs in Ise Bay, Central Japan. Bull Jpn Soc Sci Fish 1995;59(1):19-31.

Smith P, Flerx W, Hewitt R. The CalCOFI vertical egg tow (CalVET) net. pp. 27-32. Wash. DC: NOAA Technical Report NMFS 36. U.S. Dep. Commer., 1985; 
Solemdal P, Sundby S. Vertical distribution of pelagic fish eggs in relation to species, spawning behaviour and wind conditions. ICES CM 1981;pp. $1-27$.

Somarakis S, Palomera I, Garcia A, Quintanilla L, Koutsikopoulos C, Uriarte A, et al. Daily egg production of anchovy in European waters. ICES J Mar Sci 2004;61:944-958.

Sundby S. Turbulence and ichthyoplankton: influence on vertical distributions and encounter rates. Sci Mar 1997;61(S1):159-176.

Tanaka Y. Change in the egg buoyancy of Japanese anchovy Engraulis japonicus during embryonic development. Nippon Suisan Gakkaishi 1990; 56(1):165.

Tanaka Y. Japanese anchovy egg accumulation at the sea surface or pycnocline observations and model. J Oceanogr 1992;48:461-472.

Wallace R, Selman K. Cellular and dynamic aspects of oocyte growth in teleosts. Am Zool 1981;21:325-343. 